\title{
Vertical Electrical Sounding (Ves) For The Determination Of Under Ground Resistivity In Part Of Nigeria Wilberforce Island,Amassoma, Bayelsa State
}

\author{
Meindinyo, R.O.K.,Utuedeye .O, Adedokun I.O. \\ Department of Physics, Niger Delta University, Wilberforce Island, Amassoma, Bayelsa State, Nigeria
}

\begin{abstract}
Vertical Electrical Sounding (VES) using Schlumberger electrode configuration was employed in the determination of underground resistivity as well as to determine the thickness, depth and aquiferous zone in parts of Wilberforce Island Bayelsa State. A total of five Vertical Electrical Sounding (VES) were obtained using the Schlumberger electrode array configuration with maximum current electrode spacing of $400-500 m$. The data obtained was interpreted using computer assisted interpretation software (IPI2win) which showed that the area is composed of topsoil, clay, sand, clayey sand, sandy clay, medium coarse sand and fine coarse sand.Various geosounding curves were obtained at the various stations. The resistivity plot shows that stations in VES1,VES2, VES3, VES4 and VES5 have QQH, KH, QHA, KH and AA type of curves. Four geoelectric layers were identified in all VES station except that of VES1 and VES3 where five geoelectric layers were actually identified. The highest resistivity obtained in all the stations was $327.2 \Omega \mathrm{m}$ in layer one in VES 3 (at Igbedi) with a thickness of $1.9 \mathrm{~m}$ and a depth of $1.9 \mathrm{~m}$, and the lowest reistivity obtained was $8.1 \Omega \mathrm{m}$ in layer three in VES 4 (NDU New site) with a thickness of $21.1 \mathrm{~m}$ and a depth of $24.9 \mathrm{~m}$. The first and second aquifer layers were identified in layer four in VES5 (tantuama-NDU) and layer five in VES3(Igbedi) with resistivity value of $134.6 \Omega \mathrm{m}$ and $120.0 \Omega \mathrm{m}$ and depth of $100.7 \mathrm{~m}$ and $84.8 \mathrm{~m}$. Analysis of this layers shows that they compose of fine coarse sand and viable water formation. Hence, Vertical Electrical Sounding can be said to be a good alternative for the determination of underground resistivity.
\end{abstract}

Keywords: Resistivity, Underground Water, VES, Porosity, Sand Formation.

\section{Introduction}

Electrical resistivity is a geophysical method in which an electrical current is injected into the ground through steel electrodes in an attempt to measure the electrical properties of the subsurface.

Most soils and non-ore bearing rocks are electrically resistive, (i.e, insulators). Soil moisture and ground water are often electrically conductive due to contained dissolved minerals. Therefore the resistivity measured in the ground is predominantly control by the amount of moisture and water within the soil and rock (a function of the porosity and permeability), and the concentration of dissolved solids (salts) in that water. Electrical resistivity surveys have been used for many decades in hydrogeological, mining and geotechnical investigations. More recently, it has been used for environmental surveys. Resistivity surveying is commonly used for groundwater investigations. The usual practice in resistivity surveying technique is to pass current into the ground by means of two electrodes called current electrodes and to measure potential drop through a second pair of electrodes called potential electrodes. The electrodes has practically zero resistance because it is a metallic body. The principle of operation depends on the fact that any subsurface variation in conductivity alters the form of current flow within the Earth and this in turn affects the distribution of electric potential. The resistivity of common rocks, soil materials and chemicals (Keller and Frischknecht 1966, Daniel and Alberty 1966, Telford et al. 1990). Igneous and metamorphic rocks typically have high resistivity values. The resistivity of these rocks is greatly dependent on the degree of fracturing, and the percentage of the fractures filled with ground water. Thus a given rock types can have a large range of resistivity, from about 1000 to 10 million $\Omega . M$, depending on whether it is wet or dry. Usually this characteristic is useful in the detection of fracture zones and other weathering features, such as in engineering and groundwater surveys. Sedimentary rocks, which are usually more porous and have higher water content, normally have resistivity values compared to igneous and metamorphic groundwater varies from 10 to $100 \Omega$.M depending on the concentration of dissolved salts. Note the low resistivity (about $0.2 \Omega$.M) of seawater due to the relatively high salt content. This make the resistivity method an ideal technique for mapping the saline and fresh water interface in coastal areas.

Geophysical resistivity techniques is extensively used for a wide variety of geotechnical and ground water exploration problems, this is due to the fact that the electrical resistivity survey is one of the simplest and cost effective geophysical survey employed. The vertical electrical sounding (VES) is a very convenient nondestructive method of determining the depth to rock head for foundation purposes and also provides information on the degree of saturation of the subsurface materials. The vertical resistivity method has proved useful and 
successful in detecting water bearing formation in Egoro-Amede, Ekpoma south-south Nigeria (Ezomo and Ifideli, 2004). Five schlumberger vertical electrical sounding (VES) were carried out by (K.S Okiongbo and G.Ogobiri, 2011) in part of Bayelsa state Nigeria to investigate groundwater resources in Bayelsa state and the result of the study shows a laterally and vertically high and low resistive geoelectrical layers throughout the whole areas. This indicate sandy formation with intercalation of clay bodies which is typical of complex depositional environment of the area. (Fuller and Russell, 1956) carried out similar work at the northern part of the state of mission in California to partially localize and determine the thickness and depth of both near surface and basal fresh water bearing gravel bodies in glacial deposit and came up with the conclusion that larger amount of portable water are contained in gravel deposit at the base of the glacial drift and the glacial stream channels, the gravel can be as much as 50M thick. (Narachnkun, 1999) carried out electrical resistivity in the Niger Delta region and concluded from its result that the water table is shallow at about $0.3 \mathrm{M}$ in most places and as such deep boreholes of $350-600 \mathrm{M}$ and above are recommended to obtain portable and better quality groundwater since low $\mathrm{PH}$, high iron concentration, high manganese concentration and salt water encroachment has posed a problem to groundwater quality in the study area.

\section{Theory of Electrical Resistivity Survey.}

Electrical methods employ an artificial source of current which is introduced into the ground either by direct current or sinusoidal flow of steady (low frequency alternating current (A.C) located to the exterior and the potential difference is measured through another pair of electrodes called the potential electrode.

\section{Ohm's Law and Resistivity}

In a homogeneous earth, current flows radially outward from the source to define a hemispherical surface. The current distribution is equal everywhere on this surface which is also called an equipotential surface. Starting with ohm's law

$$
\mathrm{V}=\mathrm{IR}
$$

And defining the resistance $\mathrm{R}$ in terms of the resistivity $\mathrm{P}$ and the area of the shell (equipotential surface), the potential difference across the shell is

$$
d v=i(R)=I\left(\ell \frac{L}{A}\right)=I\left(\ell \frac{d r}{2 \pi r^{2}}\right)
$$

Where:

$\mathrm{V}=$ is the voltage (or electrical potential),

$\mathrm{I}=$ is the current,

$\ell=$ is the resistivity,

$r=$ is the radius of the equipotential surface.

Integrating the above equation and setting the potential at infinity to zero, the electric potential at a distance $\mathrm{R}$ from the source is given by

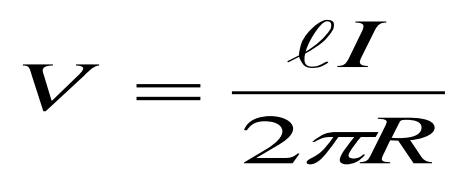

Resistivity has units of ohm M and is not to be confused with resistance which has units of ohms. The resistivity of a material is defined as

$$
\ell=\frac{R A}{L}
$$

Where:

$\mathrm{R}=$ is the resistance of the material,

A $=$ is the cross-sectional area through which current flows,

$\mathrm{L}=$ is the length on the material. 
The potential has been derived due to a single current source. The goal in resistivity surveying is to measure the potential difference between two points due to the current from two current electrodes. The potential at each electrode is determined due to the current sources;

$$
\begin{aligned}
& V \ell_{1}=\frac{I \ell}{2 \pi r_{1}}-\frac{I \ell}{2 \pi r_{2}} \\
& V \ell_{2}=\frac{I \ell}{2 \pi r_{3}}-\frac{I \ell}{2 \pi r_{4}}
\end{aligned}
$$

The potential difference $\Delta \mathrm{V}=\mathrm{V} \ell_{1}-\mathrm{V} \ell_{2}$ which simplifies to

$$
\Delta V=\frac{I \ell}{2 \pi}\left(\frac{1}{r_{1}}-\frac{1}{r_{2}}-\frac{1}{r_{3}}+\frac{1}{r_{4}}\right)
$$

The above equation can then be solved for the resistivity P. In a non homogeneous earth, the resistivity which is measured is not actually the true resisitivity of the subsurface. For an earth with more than one layer, the apparent resistivity measured will be an average of the resistivities of the additional layers. The apparent resistivity data needs to be interpreted in terms of a subsurface model in order to determine the actual resistivities of the layers.

\section{Apparent Resistivity}

Apparent resistivity is defined as the resistivity of an electrically homogeneous and isotropic half-space that would yield the measured relationship between the applied current and the potential difference for a particular arrangement and spacing of electrodes. An equation giving the apparent resistivity in terms of applied current, distribution of potential and arrangement of electrodes can be arrived at through an examination of the potential distribution due to a single current electrode. The effect of an electrode pair (or any other combination) can be found by superposition. Consider a single point electrode, located on the boundary of a semi-infinite, electrically homogeneous medium, which represents a fictitious homogeneous earth. If the electrode carries a current I, measured in amperes (a), the potential at any point in the medium or on the boundary is given by:

$$
U=\frac{\ell I}{2 \pi r}
$$

Where:

$\mathrm{U}=$ Potential in $\mathrm{V}$,

$\ell=$ Resistivity of the medium,

$r=$ Distance from the electrode.

For an electrode pair with current I at electrode A, and -I at electrode B (Fig.1), the potential at a point is giving by the algebraic sum of the individual contributions:

$$
U=\frac{\ell I}{2 \pi r_{A}}-\frac{\ell I}{2 \pi r_{B}}=\frac{\ell I}{2 \pi}\left[\frac{1}{r_{A}}-\frac{1}{r_{B}}\right]
$$

Where $r_{A}$ and $r_{B}=$ distance from the point to electrodes A and B. Fig(1) illustrate the electric field around the two electrodes in terms of equipotentials and current lines. The equipotentials represent imagery shells, or bowls surrounding the current electrodes and on any one of which the electrical potential is everywhere equal. The current line represent a sampling of the infinitely many paths followed by the current, paths that defined by the condition that they must be everywhere normal to the equipotential surfaces. 


\section{Schlumberger Array}

The schlumberger array (Fig. 2.2), consists of four collinear electrodes. The outer two electrodes are current (source) electrodes and the inner two electrodes are the potential (receiver) electrodes. The potential electrodes are installed at the center of the electrode array with a small separation, typically less than one fifth of the spacing between the current electrodes. The current electrodes are increased to a greater separation during the survey while the potential electrodes remain in the same position until the observed voltage becomes too small to measure. Typically, expanding the current electrodes occurs roughly six(6) times per decade. The advantages of the schlumberger array are that fewer electrodes need to be moved for each sounding and the cable length for the potential electrodes is shorter. Schlumberger sounding generally have better resolution, greater probing depth, and less time-consuming field deployment than the wenner array. The disadvantages are that long current electrode cables are required, the recording instrument needs to be very sensitive, and the array may be difficult or confusing to co-ordinate amongst the field crew.

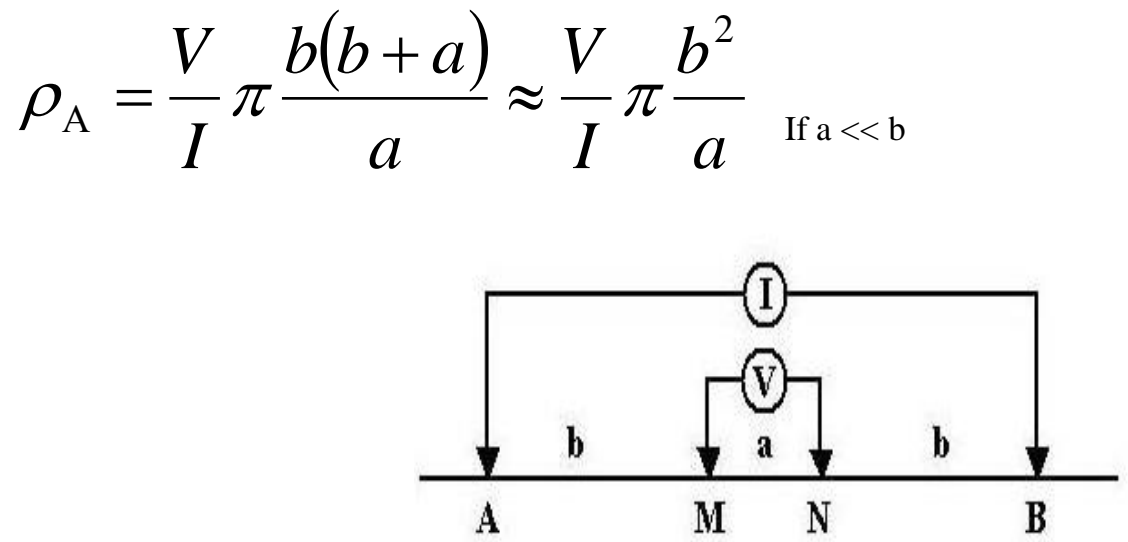

Fig.1: Schlumberger Array

\section{Methodology}

The electrical resistivity varies between different geological materials, depend s mainly on variation in water contents and dissolved ion in the water. Resistivity can thus be used to identify zones with different electrical properties, which can then be referred to different geological strata. Resistivity is also called specific resistance which is the inverse of conductivity or specific conductance. The practice of this method in the field is to apply an electrical direct current (DC) through two electrodes driven into the ground knows as current electrode, and to measure the resulting potential difference of an additional electrode knows as the potential electrodes, the current used is either direct current or alternative current of low frequency about $20 \mathrm{~Hz}$. Materials used were Abem Terrameter, Electrode (current and potentials), Rechargeable battery, Measuring Tape, Cables, Hammer, Global positioning system (GPS), Recording, Sheet.

\section{Data Acquisition}

In this geophysical resistivity technique, the vertical electrical sounding (VES) using the schlumberger electrode array configuration was employed. A total of five (5) schlumberger vertical electrical sounding was carried out in an area with array spread for current electrodes spacing ranging between 400-500. In the VES method of survey current is driven into the ground using the current electrodes $C_{1}$ and $C_{2}$ and the resulting potential is measured using the two potential electrodes $\ell_{1}$ and $\ell_{2}$, the potential electrode remain fixed at a point while the current electrodes are expanded symmetrically about the centre of the spread; The potential electrodes was also increased at a large value of the current electrode spread to ensure that the distance between $\ell_{1}$ and $\ell_{2}$ never exceeds $\mathrm{AB} / 2$ and $\mathrm{AB}$ is the distance between current electrodes. The value of the resistance is measured by the ABEM Terrameter and the apparent reisistivity calculated by multiplying the geometry factor $\mathrm{K}$ with the resistance measured by the terrameter relationship is given below.

$\ell=\mathrm{KR}$

Where,

$\mathrm{K}=$ Geometric factor

$\mathrm{R}=$ Resistance

$\ell=$ Apparent resistivity.

The following were necessary precaution taken during the VES survey: 
1. The electrode was driven into the ground at great depth to avoid negative readings.

2. During the survey the ABEM Terrameter was not kept under the sun or rain when reading was taken.

\section{Results And Discussion}

Geoelectrical investigation involving Vertical Electrical Sounding (VES) were conducted to have foreknowledge on under ground resistivity. In analyzing the data the apparent resistivity was determine by multiplying the resistance measured by the terrameter and the geometric factor $\mathrm{K} 1$ the geometric factor is a parameter which is dependent on the potential and current electrode spacing.

The field curve was initially made by plotting apparent resistivity against $\mathrm{AB} / 2$ using the IPI2WIN (ID resistivity interpretation software) this was used to smoothen the curve and the data obtained from this was later subjected to computer assisted iterated interpretation using 1-D inversion technique software (1XID, interpex, USA) and IPI2win. This programme was used to perform qualitative analysis and interpretation of the field curves, the software requires that the operator introduces the number, thickness and resistivity of the sublayers. The theoretical curve for the initial parameters is compared with the measured data. The starting model and its resistivity are transformed and refined by the programme to obtain a best fit relation to the field data. The method or iteration was performed until the error between the field data and the synthetic model became least and constant. Data obtained from the survey and their corresponding model curves are shown below.

Five geosounding was carried out at locations in some parts of Wilberforce Island, Bayelsa state. The results of the geosounding curves and their interpretations are shown below.

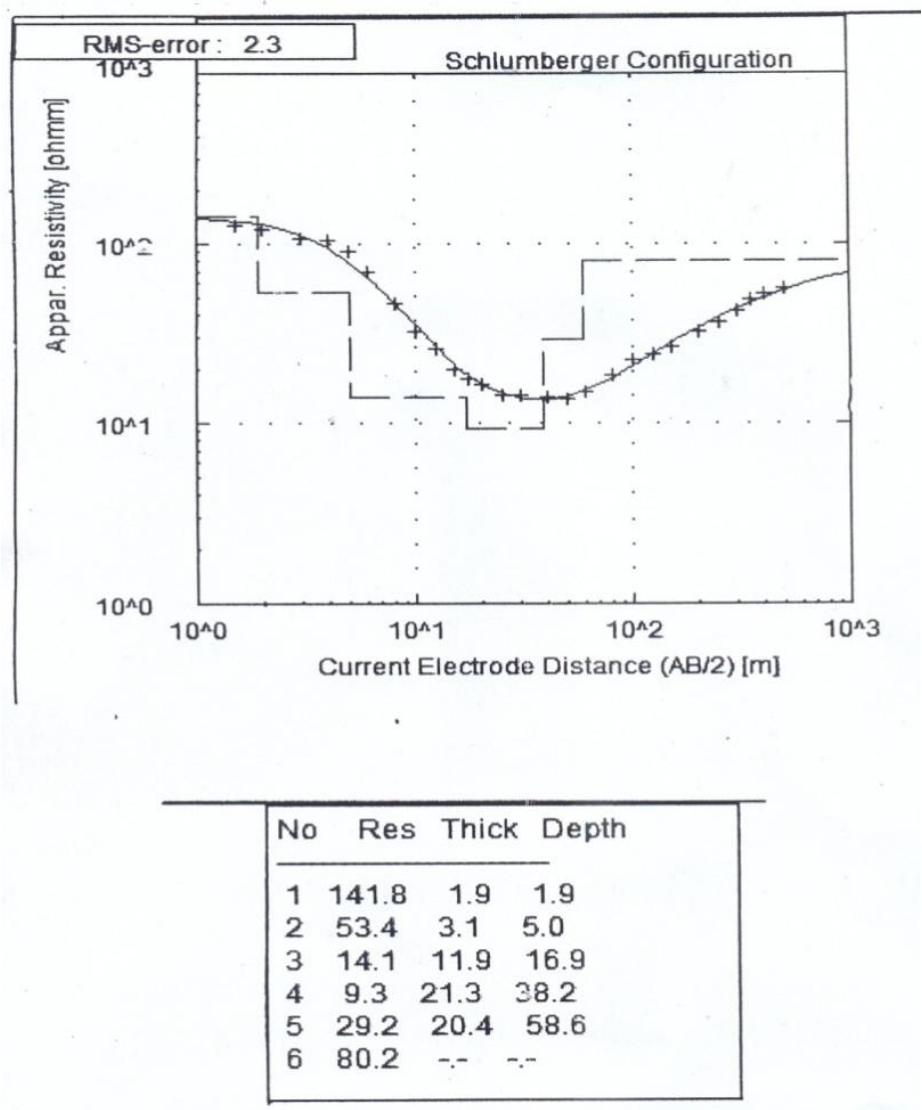

Fig.2: Geoelectric layers, field and theoretical curves for VES 1 


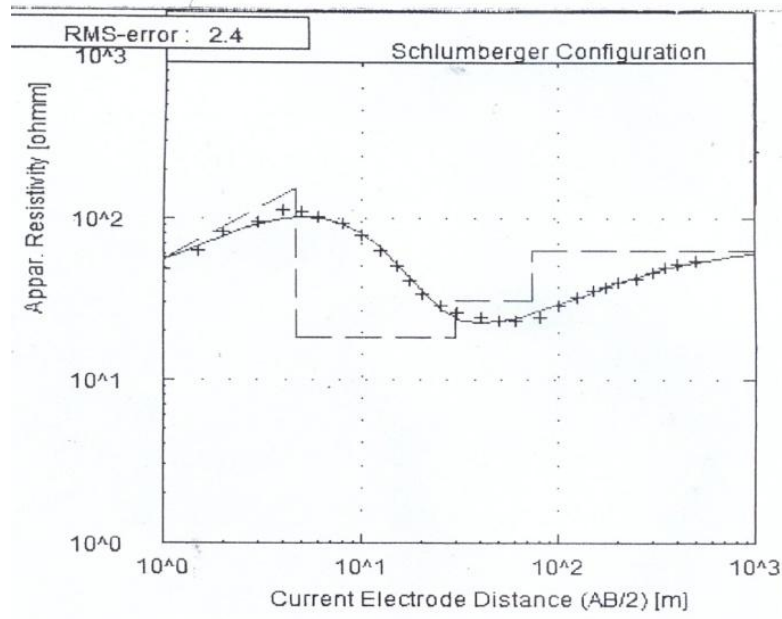

$\mid$\begin{tabular}{|cccc}
\hline \hline No & Res & Thick & Depth \\
\cline { 2 - 5 } 1 & 47.9 & 0.8 & 0.8 \\
2 & 154.2 & 3.8 & 4.6 \\
3 & 18.2 & 25.1 & 29.7 \\
4 & 31.2 & 42.9 & 72.5 \\
5 & 63.7 &.-- &.-- \\
\hline
\end{tabular}

Fig.3: Geoelectric layers, field and theoretical curves for VES 2

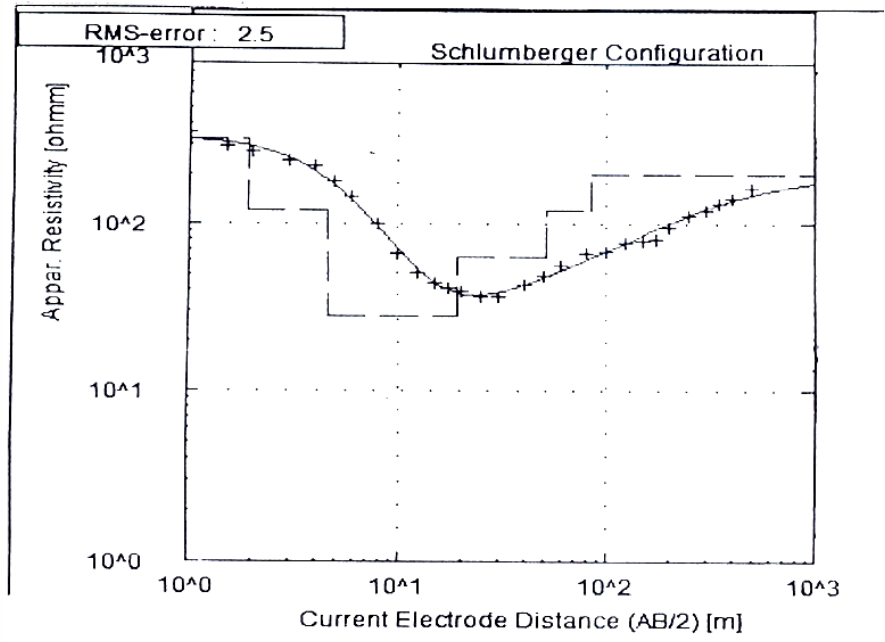

\begin{tabular}{|lccc|}
\hline No & Res & Thick & Depth \\
\hline 1 & 327.2 & 1.9 & 1.9 \\
2 & 122.0 & 2.8 & 4.6 \\
3 & 28.1 & 14.4 & 19.0 \\
4 & 63.2 & 32.0 & 51.0 \\
5 & 120.0 & 33.8 & 84.8 \\
6 & 200.2 &.-- &.-- \\
\hline
\end{tabular}

Fig.4: Geoelectric layers, field and theoretical curves for VES 3 


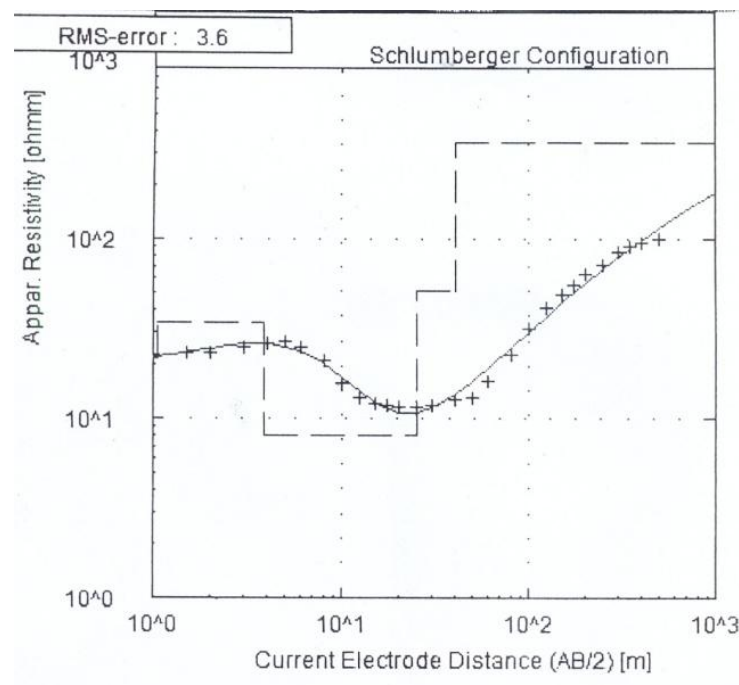

\begin{tabular}{lllll}
\hline No & Res & Thick & Depth \\
\cline { 2 - 5 } 1 & 21.5 & 1.0 & 1.0 \\
2 & 33.8 & 2.8 & 3.8 \\
3 & 8.1 & 21.1 & 24.9 \\
4 & 51.0 & 14.8 & 39.7 \\
5 & 344.7 &.-- & --
\end{tabular}

Fig.5: Geoelectric layers, field and theoretical curves for VES 4

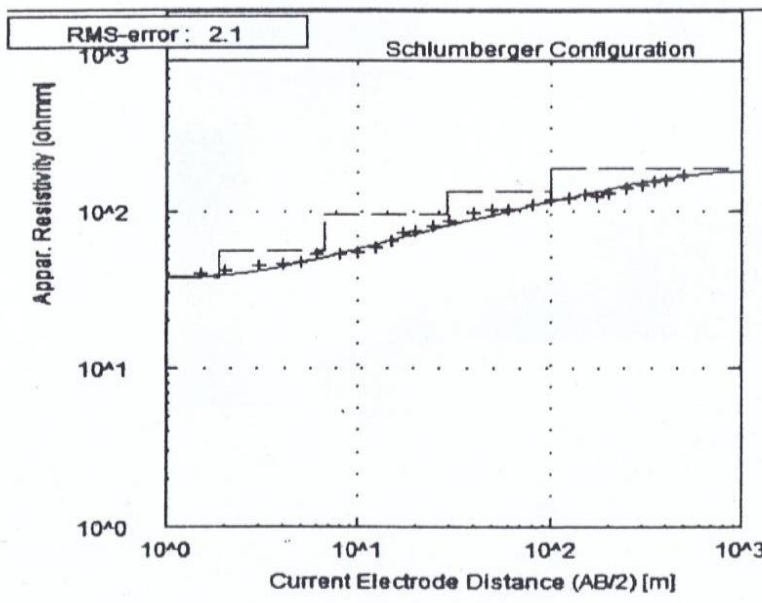

\begin{tabular}{lllll} 
No & Res & Thick Depth \\
\hline 1 & 37.8 & 1.9 & 1.9 \\
2 & 56.3 & 4.8 & 6.7 \\
3 & 94.5 & 22.6 & 29.3 \\
4 & 134.6 & 71.4 & 100.7 \\
5 & 185.6 &.-- & --
\end{tabular}

Fig.6: Geoelectric layers, field and theoretical curves for VES 5 
VES 1

In this station five geoelectric layers were identified,(Igbedi West 2). Using computer assisted interpretation (IPI2win) on the data, the geosounding curve will be of the QQH-type $\left(\ell_{1}>\ell_{2}>\ell_{3}>\ell_{4}<\ell_{5}\right)$. The topsoil which is the first layer has a resistivity of $141.8 \Omega \mathrm{m}$ and a thickness of $1.9 \mathrm{~m}$. The high resistivity indicates the presences of small amount of water and sand, so the possible lithology is wet sandy clay. The second layer has a resistivity of $53.4 \Omega \mathrm{m}$ and a thickness of $3.1 \mathrm{~m}$, with the amount of the resistivity measured, it shows that the layer is conductive which indicates the presences of a high amount of clay. The third layer has a resistivity of $14.1 \Omega \mathrm{m}$ and a thickness of $11.9 \mathrm{~m}$. The lithology here can also be said to be that of a clayey sand. The fourth layer has a resistivity of $9.3 \Omega \mathrm{m}$ and a thickness of $21.3 \mathrm{~m}$. Due to the low resistivity in this layer the lithology here is clay. The fifth layer having a resistivity of $29.2 \Omega \mathrm{m}$ and a thickness of $20.4 \mathrm{~m}$ will have a formation of clay mainly. From the table above one can noticed that there was a decrease in resistivity with increase in depth, though later increases in the fifth layer.

\section{VES 2}

In this station four geoelectric layers were identified (Igbedi East1). Using computer assisted interpretation (IPI2win) on the data, the geosounding curve will be of the KH-type $\left(\ell_{1}<\ell_{2}>\ell_{3}<\ell_{4}\right)$. The first layer which consist of the topsoil has a resistivity of $47.9 \Omega \mathrm{m}$ and a thickness of $0.8 \mathrm{~m}$. The second layer has a resistivity of $154.2 \Omega \mathrm{m}$ and a thickness of $3.8 \mathrm{~m}$. Due to the high resistivity of this layer, it can be said to be of sand formation basically sandy clay. The third layer has a resistivity of $18.2 \Omega \mathrm{m}$ and a thickness of $25.1 \mathrm{~m}$. because of its high conductivity (low resistivity), the possible lithology of the layer can be said to be that of clayey sand. The fourth layer has a resistivity of $31.2 \Omega \mathrm{m}$ and a thickness of $42.9 \mathrm{~m}$. The formation in this layer will be clay mainly. From the table above one can see that the lowest resistivity was $18.2 \Omega \mathrm{m}$ which is the third layer and has a depth of $29.7 \mathrm{~m}$.

\section{VES 3}

In this station five geoelectric layers were identified (Igbedi). Using computer assisted interpretation (IPI2win) on the data, the geosounding curve will be of the QHA-type $\left(\ell_{1}>\ell_{2}>\ell_{3}<\ell_{4}<\ell_{5}\right)$. The first layer which consist of the topsoil has a resistivity of $327.2 \Omega \mathrm{m}$ and a thickness of $1.9 \mathrm{~m}$. Due to the high resistivity of this layer it can be said to be of sand formation. To say the first layer has the highest resistivity with a depth of $1.9 \mathrm{~m}$. The second layer has a resistivity of $122.0 \Omega \mathrm{m}$ and a thickness of $2.8 \mathrm{~m}$, the high resistivity of this layer shows that it comprises of sand especially clayey sand. The third layer has a resistivity of $28.1 \Omega \mathrm{m}$ and a thickness of $14.4 \mathrm{~m}$,because of the high conductivity (low resistivity), the lithology here will be of that of clay. The fourth layer has a resistivity of $63.2 \Omega \mathrm{m}$ and a thickness of $32.0 \mathrm{~m}$, with the amount of resistivity measured it shows that the layer is conductive which indicates the presence of a high amount of clay. The fifth layer has a resistivity of $120.0 \Omega \mathrm{m}$ and a thickness of $33.8 \mathrm{~m}$, the high resistivity of this layer shows that it comprises of sand especially medium coarse sand. This is also likely an aquiferous layer. Infact its water quality should be better than that of the fourth layer and it also have a thickness greater than that of the fourth layer.

\section{VES 4}

In this station four geoelectric layers were identified(at NDU new site). Using computer assisted interpretation (IPI2win) on the data, the geosounding curve will be of the KH-type $\left(\ell_{1}<\ell_{2}>\ell_{3}<\ell_{4}\right)$. The topsoil which is the first layer has a resistivity of $21.5 \Omega \mathrm{m}$ and a thickness of $1.0 \mathrm{~m}$. The second layer having a resistivity of $33.8 \Omega \mathrm{m}$ and a thickness of $2.8 \mathrm{~m}$ will have a formation of clay mainly. The third layer has a resistivity of $8.1 \Omega \mathrm{m}$ and a thickness of $21.1 \mathrm{~m}$, the low resistivity which indicates high conductivity suggest that the formation of this layer is mainly clay. The fourth layer has a resistivity of $51.0 \Omega \mathrm{m}$ and a thickness of $14.8 \mathrm{~m}$, with the amount of the resistivity measured it shows that the layer is conductive like the third layer so, the lithology here can also be said to be that of clayey sand. From the table above layer three has the lowest resistivity of $8.1 \Omega \mathrm{m}$ with a depth of $24.9 \mathrm{~m}$ while layer four has the highest resistivity of $51.0 \Omega \mathrm{m}$ with a depth of $39.7 \mathrm{~m}$.

\section{VES 5}

In this station four geoelectric layers were identified (around Tantuama-NDU). Using computer assisted interpretation (IPI2win) on the data, the geosounding curve will be of the AA-type $\left(\ell_{1}<\ell_{2}<\ell_{3}<\ell_{4}\right)$. The first and second layers which consist of the topsoil has a resistivities of $37.8 \Omega \mathrm{m}, 1.9 \mathrm{~m}$ and $56.3 \Omega \mathrm{m}, 4.8 \mathrm{~m}$. Due to the low resistivity (high conductivity) which indicates the presences of a high amount of clay. The third layer has a resistivity of $94.5 \Omega \mathrm{m}$ and a thickness of $22.6 \mathrm{~m}$, the resistivity here suggest that the formation of this layer will be that of sandy clay. The fourth layer has a resistivity of $134.6 \Omega \mathrm{m}$ and a thickness of $71.4 \mathrm{~m}$, the high resistivity of this layer shows that it comprises of sand especially fine coarse sand. This is also likely an aquiferous layer. 


\section{Summary}

From the above interpretations of the VES we can say that in all the VES stations, it can be noticed that four(4) geoelectric layers were actually identified in all stations except station one(1) and three(3) where five(5) geoelectric layers were identified.

In VES1, (Igbedi west 2) the resistivity was decreasing with an increase in depth, though later increases in the fifth layer.

In VES2, (Igbedi east 1) layer two has the highest resistivity with low thickness of $3.8 \mathrm{~m}$.

In VES3, (igbedi) layer one has the highest resistivity with increase in depth.

In VES4, (NDU new site) layer four has the highest resistivity, which shows that the layer is conductive and the lithology in this layer is clayey sand.

In VES5, (Around Tantuama - NDU) the resistivity was increasing from the range of $37.8 \Omega \mathrm{m}$ to $134.6 \Omega \mathrm{m}$ as well as increase in depth.

In all the VES stations we can notice that the highest resistivity obtained was $327.2 \Omega \mathrm{m}$ in layer one in VES3 (At Igbedi) it has a thickness of $1.9 \mathrm{~m}$ and a depth of $1.9 \mathrm{~m}$ as well. And the lowest resistivity obtained was $8.1 \Omega \mathrm{m}$ in layer three VES4 (NDU New site) it has a thickness of $21.1 \mathrm{~m}$ and a depth of $24.9 \mathrm{~m}$.

From the above analysis, it is evident how Vertical Electrical Sounding plays a major role in determining underground resistivity.

\section{Conclusion}

Five Vertical electrical soundingwere carried out in five different locations in parts of Wilberforce Island Bayelsa State. The highest resistivity obtained in all the stations was $327.2 \Omega \mathrm{m}$ in layer one in VES 3 (at Igbedi) with a thickness of $1.9 \mathrm{~m}$ and a depth of $1.9 \mathrm{~m}$, and the lowest resistivity obtained was $8.1 \Omega \mathrm{m}$ in layer three in VES 4 (NDU New site) with a thickness of $21.1 \mathrm{~m}$ and a depth of $24.9 \mathrm{~m}$. The first and second aquifer layers were identified in layer four in VES5 (Tantuama-NDU) and layer five in VES3 (Igbedi) with resistivity value of $134.6 \Omega \mathrm{m}$ and $120.0 \Omega \mathrm{m}$ and depth of $100.7 \mathrm{~m}$ and $84.8 \mathrm{~m}$. Analysis of this layers shows that they comprises of fine coarse sand and viable water formation. Hence, Boreholes for potable groundwater are therefore recommended within the fourth and fifth layers in VES 5 (Tantuama NDU) and VES 3 (Igbedi). Vertical Electrical Sounding can be said to be a good alternative for the determination of underground resistivity.

\section{References}

[1]. Okiongbo, Kenneth S, (2012). Determination of aquifer properties and ground Water vulnerability mapping using geoelectrical Method in Yenagoa City.Ako, B. D. (1982), Geophysical prospecting for ground water in an area adequate geological data base'. J. Min. Geology; 18: 88 -150.

[2]. Aseez, L. O. (1989) 'Review of the stratigraphy, sedimentation, and structure of the Niger Delta' Rocks and stone (Nig) Ltd Ibandan Nigeria. Geology of Niger Delta Edited by C.A. Kogbe;3: 16 - 318.

[3]. Lowrie, W. (2004). 'Fundamental of Geophysics' published by Cambridge university press 4:212-218

[4]. Mailet, R. (1974)" The fundamental equation of electrical prospecting' vol. 12, .529-556.

[5]. Okiongbo, K. S. and Ogobiri, G. (2011)' Geoelectrical investigation of ground water in Bayelsa state, Nigeria. 'Research journal of environmental and earth science, 3(6);620-624.

[6]. Osemefichian, J. E. A. and Asokhia, M. B. (1994) 'Applied geophysics' published by samtos service limited Lagos state Nigeria. 7:96-111.

[7]. Reynolds, J. M. (1997). An introduction to applied and environmental geophysics'ISI3N 0.471.95555-8, Johnwlley and sons, chicester,796.

[8]. Tattam, C. M. (1937), The application of electrical resistivity prospecting to groundwater problem' Colorado school of mines; journal of geophysical students, vol. $32: 1$ 17-120.

[9]. Telford, W. M., Geldart, L. P. and Sheriff, R. E. (1990) 'Applied geophysics, $2^{\text {nd }}$ edition' Cambridge university press; 770

[10]. Wightman, W. E. F., Jalinoos, Sirles, P. (2003), Application of geophysical Problem to high way problems.’ Federal highway Administration, Central federal Lands Highway Division, Lakewood. :1-11.

[11]. Wightman, W.E., Jalinoos, F., Sirles, P., and Hanna, K. (2003). "Application of Geophysical Methods to Highway Related problem." Federal Highway Administration, Central Federal Lands Highway Division, Lakewood, CO, Publication No. FHWA-IF04-021, September 2003

[12]. Kelly, (1966). "Dipole Method for Deep Resistivity studies.

[13]. EPA, (1993) "Use of Airborne, Surface, and Borehole Geophysical Techniques at Contaminated Sites".

[14]. Morrison and Gasperikkova,(2012) "DC Resistivity and IP field systems, data processing ansd interpretation". 